\title{
El alcoholismo como manifestación de goce autoerótico en un sujeto adicto: Julio Luna
}

\author{
Alcoholism as a manifestation of autoerotic enjoyment in an addicted subject: \\ Julio Luna
}

Alcoolismo como manifestação de gozo autoerótico em um sujeito viciado: Julio Luna

\author{
Ilias Wakkas \\ Ilias_8@ hotmail.com \\ https://orcid.org/0000-0003-3558-6219
}

Universidad Arturo Michelena, Valencia, Venezuela

RESUMEN

El alcohol se presenta en el adicto como una sustancia que le proporciona sensaciones biológicas, psicológicas y sociales que el mismo sujeto encamina hacia el encuentro con la carencia que es causa de su sufrimiento. La investigación tuvo como propósito develar desde donde se asume el alcoholismo como manifestación de goce autoerótico en el sujeto adicto, partiendo de la historia de vida de Julio Luna. Su metodología fue cualitativa; con un diseño cualitativa; bajo el método historia de vida; las fases de la investigación fueron prehistoria, la historia y la interpretación, los elementos de la historia-de-vida fueron los bloques-de-sentido y las marcasguías. Como resultado se obtuvo, el alcohol para el sujeto adicto proporciona un modo de gozar en su propio cuerpo bajo las premisas autoeróticas. Finalizando, el alcohol, soslaya la falta inmersa del ser $y$, por ende, el alcoholismo como síntoma adquiere forma y relevancia a partir de este goce.

Palabras clave:

Alcoholismo; autoerótico; sujeto adicto, historia de vida
ABSTRACT

RESUMO

Alcohol appears in the addict as a substance that provides him with biological, psychological and social sensations that the same subject leads towards the encounter with the lack that is the cause of his suffering. The purpose of the research was to reveal from where alcoholism is assumed as a manifestation of autoerotic enjoyment in the addicted subject, starting from the life story of Julio Luna. His methodology was qualitative; with a qualitative design; under the life history method; the phases of the investigation were prehistory, history and interpretation, the elements of the history-of-life were the blocks-of-sense and the marks-guides. As a result, it was obtained, alcohol for the addicted subject provides a way to enjoy his own body under autoerotic premises. Finally, alcohol ignores the immersed lack of being and, therefore, alcoholism as a symptom acquires shape and relevance from this enjoyment.

Key words:

Alcoholism; autoerotic; addicted subject, life story
O álcool aparece no dependente químico como uma substância que the proporciona sensações biológicas, psicológicas e sociais que o próprio sujeito direciona para o encontro com a carência que the causa sofrimento. O objetivo da pesquisa foi revelar de onde - alcoolismo é assumido como manifestação de gozo autoerótico no sujeito dependente, a partir da história de vida de Julio Luna. Sua metodologia era qualitativa; com design qualitativo; sob o método da história de vida; as fases da investigação foram a pré-história, a história e a interpretação; os elementos da história de vida foram os blocos de sentido e os marcos. Como resultado, foi obtido que o álcool para o sujeito viciado oferece uma forma de desfrutar o próprio corpo sob premissas autoeróticas. Por fim, o álcool ignora a falta de ser imersa e, portanto, o alcoolismo como sintoma adquire forma e relevância a partir desse gozo.

Palavras-chave:

Alcoholism; autoerótico; assunto viciado; história de vida 


\section{INTRODUCCIÓN}

El alcohol y la historia del mismo en el mundo, se presentan desde épocas remotas con el descubrimiento de la fermentación y, aunado a ello, a los avances tecnológicos que contribuyeron al desarrollo y fabricación de productos y licores en la actualidad. Sin embargo, el alcohol tiene bases ancestrales, es decir, antes del cristianismo, por ejemplo, en el banquete de Platón. Respecto a la prehistoria del alcoholismo, Sarmiento (2011) plantea que:

El alcohol, del árabe alkuhi «esencia o espíritu», es la droga más consumida en el mundo. Su uso en forma de brebaje, se supone que data de los albores de la humanidad y la ingestión inicial, vinculada con actividades religiosas, dio paso a su consumo colectivo (p. 1).

Por ende, la historia del alcohol se remonta a la época del hombre primitivo, donde los mismos descubrieron que podían conservar el producto de sus cosechas mediante la fermentación, transformándolos en bebidas alcohólicas.

Pero para esta época no existía la transformación tecnológica y el capitalismo actual, por ello, las bebidas derivadas de la fermentación eran en su mayoría de uso religioso. Los inicios de la fabricación del alcohol a base de fermentación para su uso colectivo se remontan a Pasteur (1880), padre de la microbiología, quien consiguió explicar la fermentación.

Considerando esto, la entrada del alcohol al discurso capitalista y consumo en el mercado tiene como principal sucesor al francés Pasteur (1880), teniendo esto como consecuencia la masificación e industrialización del alcohol y su ingesta ya no como uso religioso, sino más bien como producto del mercado que ofrece las mejores garantías de una satisfacción plena e inmediata.
A su vez, la constitución del alcohol adquiere relevancia en la contemporaneidad. Por ello, Lora y Calderón (2010) afirman que “(...) la búsqueda de nuevas experiencias sensitivas y la alteración de la conciencia se observa en registros arqueológicos antiquísimos. Sin embargo, hasta la época contemporánea, no aparecieron como problema para las diferentes culturas" (p. 152). El problema cultural del alcohol aparece entonces luego de su embotellamiento y comercialización en el mercado y, entonces, son creadas las leyes contra el consumo excesivo del alcohol para contrarrestar los daños que causa el mismo en las sociedades, como, por ejemplo, los niveles de mortandad.

La problemática del alcoholismo ha sido de suficiente interés por parte de entes gubernamentales, considerando sus implicaciones en la sociedad como los daños físicos por causa del mismo.

Países como en Venezuela, es donde se presentan los mayores índices de muerte por causa del alcohol. En cuanto a índices, Díaz (2017) anuncia que "las muertes de hombres en Venezuela, generadas por el alcohol, obtienen una cifra de 96,6 fallecimientos por cada 100 mil habitantes y en las mujeres unos 6,2 fallecimientos por cada 100 mil" (p. 1) De esta manera, es claro que el consumo masivo del alcohol conlleva consecuencias marcadas en el ámbito social y cultural.

Por consiguiente, las ciencias médicas y clínicas han hecho grandes contribuciones al fenómeno del alcoholismo, pero han hecho de todas estas contribuciones unos hechos generalizados $y$ estandarizados, aplicables para todo ser hablante, perdiendo el punto de vista más importante de la postmodernidad; lo singular y la subjetividad. Aunado a ello, (Zafiropoulos 1988, citado en Cifuentes, 2015) indica que: 
De esta manera, las perspectivas hegemónicas sobre «la adicción» difundidas por el discurso médico y psicológico bajo su concepción de «enfermedad» o «trastorno» han definido la problemática desde discursos homogeneizantes, transmisores demensajesideológicos, morales, políticos, que soslayan la pregunta por la complejidad del fenómeno y el recorrido particular de cada sujeto (p. 22).

Todas estas contribuciones de la ciencia moderna dan testimonio de los innumerables intentos por dar al fenómeno del alcoholismo una comprensión universal y solución al problema que se presenta por el mismo en toda la humanidad. Prácticas que no han sido otra cosa que meros intentos por ocultar o en mejores términos, de disfrazar el síntoma que no desaparece pero que si reaparece. De esto podemos dar testimonio en las continuas recaídas del adicto, trayendo como consecuencia perjudiciales condiciones más marcadas de padecimiento en el sujeto alcohólico.

Además, el goce autoerótico se presenta en el adicto como un goce que implica la ruptura con el universo simbólico. Por ello, el alcohol es un modo de goce asexual, un goce que no requiere del otro; a ello atribuimos la abstinencia sexual presente en el sujeto alcohólico, de modo que este goce trabaja en función de lo mítico, de lo imaginario, y de esta forma impidiendo el desplazamiento de la cadena de significantes que acalla el goce superyoico inconsciente. Siguiendo la idea de ruptura simbólica, Mora (2015) plantea que:

En la toxicomanía se produce una ruptura con el falo, con la función paterna que se encuentra más ligada al principio del placer. Los sujetos experimentan un goce que se sitúa más allá del hedonismo, que también está ligado a una satisfacción, pero es una satisfacción de otro orden, un exceso de ella que confluye con la pulsión de muerte (p. 2).

Considerando esto, el adicto sumergido al dominio de la pulsión de muerte y en su forma de hacerse gozar en su propio cuerpo con el deseo de ingesta de alcohol para reprimir el goce fálico que lo avergüenza, es empujado por el deseo, el exceso y destrucción.

En este sentido, la presente investigación tiene como propósito develar desde donde se asume el alcoholismo como manifestación de goce autoerótico en el sujeto adicto, partiendo de la historia de vida de Julio Luna.

La presente investigación nace como fruto de la curiosidad y añoranza de conocimiento respecto al fenómeno del alcoholismo y del sufrimiento en el sujeto adicto; de la fascinación de una problemática que se presenta en el adicto como el mejor escape al mundo simbólico; el alcoholismo que implica la satisfacción inmediata y sumerge al hombre en su propio destino: la muerte.

Se trata de un trabajo de investigación que tiene la oportunidad de desarrollar la práctica del psicoanálisis dentro de la psicología clínica. Por la misma razón se trata de una historia de vida $y$, con ello pasar de la escucha a la escritura y comprensión del síntoma y de su experiencia con el mismo. De esta manera, abordar el sujeto adicto desde el marco de la psicología, la prehistoria, el análisis y la subjetividad.

\section{El Síntoma en psicoanálisis}

Freud (1917) plantea que "los síntomas neuróticos tienen entonces su sentido, como las operaciones fallidas y los sueños, y, al igual que estos, su nexo con la vida de las personas que los exhiben" (p. 235-236). Es decir, lo inconsciente tomaría el decurso hacia la irrupción en la 
consciencia a través de cualquier rodeo, entre ellos el mismo síntoma.

Por ello, Freud le devuelve valor al síntoma a través de la cura analítica. Al adoptar la asociación libre como método catártico, el síntoma se posiciona como camino del desciframiento del inconsciente, por ello, la intención del analista no sería la abolición de dicho síntoma, sino más bien entender el mismo mediante la escucha. Freud (1917) manifiesta que "(...) el sentido de un síntoma reside según tenemos averiguado, en un vínculo con el vivenciar del enfermo" (p. 246-247). Es decir, el sentido del síntoma posee valor en la experiencia subjetiva del enfermo $y$, descifrar el mismo es sobrepasar el sin sentido propio de la consciencia.

Por ello, la hipnosis y la abreacción mediante la asociación en el decurso consciente de las representaciones estranguladas por el mecanismo de la represión, resultarían insuficientes en la cura analítica, por esto, el discurso del sujeto cobraría importancia en el desciframiento del síntoma a partir de lo que el mismo sujeto quería manifestar.

\section{La adicción desde la perspectiva lacaniana}

Para Lacan (1938), la familia es donde comienza el mismo a reformular algunas ideas freudianas del complejo primitivo para luego, plantear como llega este complejo a implicar en la adicción.

El complejo del destete fija en el psiquismo la relación de la cría, bajo la forma parasitaria exigida por las necesidades de la primera edad del hombre; representa la forma primordial de la imago materna. De ese modo, da lugar a los sentimientos más arcaicos y más estables que unen al individuo con la familia. Abordamos en este caso el complejo más primitivo del desarrollo psíquico que se integra a todos los complejos ulteriores (pp. 30-31).
Esto reformularía las ideas freudiana en cuanto a los señalamientos de las formaciones narcisistas y el autoerotismo primitivo. El fenómeno de la drogadicción, el mismo Lacan (1938), lo plantearía cuando formula que “(...) en realidad, y a través de alguna de las contingencias operatorias que comporta el destete es a menudo un trauma psíquico cuyos efectos individuales «anorexias llamadas mentales, toxicomanías por vía oral, neurosis gástrica» revelan sus causas al psicoanálisis" (p. 32). Es entonces, desde la vida arcaica del sujeto donde se fija la relación erótica con el objeto parcial de satisfacción orgánica como lo es el seno de la madre, objeto que quedaría relegado en la vida adulta por las sustancias embriagadoras.

Lacan (1972), otorgaría importancia a lo que significa el objeto «droga» para el adicto. En el toxicómano entonces, la satisfacción del deseo se vuelve una necesidad, devolviendo al hombre a su condición animal, sin aceptar postergación, ni sustitución metafórica de ningún tipo, entregado a la sustancia embriagadora sin límites como también entregado al exceso del mismo.

\section{La ruptura con el falo}

Es conveniente señalar en un principio que una de las funciones del significante padre "falo" es la regulación, por ende, la ruptura de la misma implicaría los excesos del goce sin límites propio del sujeto adicto en su relación con la sustancia adictiva. Respecto a ello, Cifuentes (2014), plantea que "desde la perspectiva lacaniana, se plantea que "la verdadera toxicomanía es aquella en la cual la droga permite romper con el falo, detonándose un exceso que genera una pérdida de la regulación fálica" (p.23). Es por esto que el adicto se encontraría entregado al autoerotismo propio, permitiéndose gozar en su propio cuerpo y sin mediación simbólica. 
Respecto al goce del adicto Miller (1989), plantea que "(...) la droga aparece como un objeto que concierne menos al sujeto de la palabra que al sujeto del goce, en tanto ella permite obtener goce sin pasar por el Otro" (p. 16). Es decir, la sustancia adictiva propicia un modo de gozar en el propio cuerpo y prescindir de la castración «falo», en tanto sujeto de la palabra. Este modo de goce es el originalmente propiciado por el sujeto en la masturbación. Respecto a ello, Rojas (2007), expresa que:

En efecto, ¿cuál es la maniobra en la toxicomanía si no la de buscar, ante todo, denunciar una unión en el campo del otro, que debe conducir al sujeto a un agarre en la función fálica? Esto trae como consecuencia ocasional el que esta formación de ruptura, que permite deshacerse del aprieto de muchas confrontaciones con el otro sexo, incluso con la alteridad, contravenga la definición del síntoma en el sentido analítico (p. 139).

La posición de rechazo que adopta el sujeto está fundada en la elección de un goce recuperado en el propio cuerpo gracias al producto.

\section{Goce autoerótico y adicción}

Freud (1897), en cuanto al autoerotismo primordial, plantea "se me ha abierto la intelección de la masturbación es el único gran hábito que cabe designar "adicción primordial», y las otras adicciones sólo cobran vida como sustitutos y relevos de aquella (el alcoholismo, morfinismo, tabaquismo, etc.)" (p. 314). Por ello, el erotismo primordial del infante constituiría a la masturbación como centro de las primeras etapas del desarrollo evolutivo del sujeto.

En este sentido, a las sustancias adictivas se les deben la ganancia inmediata de placer. Es decir, a esto se refiere que el objeto droga permitiría al adicto propiciarse un goce que no requiere del otro, en tanto un goce narcisista y sustitutivo de la adicción primordial "la masturbación". Freud (1898) argumenta que:

(...) Una indagación más precisa
demuestra por lo general que esos
narcóticos están destinados a sustituir
- de manera directa o mediante unos
rodeos-el goce sexual faltante, y
cuando ya no se pueda restablecer una
vida sexual normal, cabrá esperar con
certeza la recaída del deshabituado ( $\mathrm{p}$.
268).

De ese modo, si el objeto droga implica para el toxicómano la sustitución del goce sexual faltante, no sería otro goce que aquel goce primitivo de las satisfacciones más arcaicas de la vida infantil. De esta manera, el adicto entregado a la compulsión de ingerir la bebida y, entregado a ella sin límites, goza sin necesidad de la relación sexual, goza sin pasar por el otro y con ello, el eclipse narcisista del yo propicia la abolición del universo simbólico.

Plantea Freud (1920) que “(...) la pulsión es el elemento más importante y oscuro de la investigación psicológica" (p. 34). Respecto a la pulsión, el mismo autor plantea que:

\begin{abstract}
Una pulsión sería entonces un esfuerzo, inherente a lo orgánico vivo, de reproducción de un estado anterior que lo vivo debió resignar bajo el influjo de fuerzas perturbadoras externas; sería una suerte de elasticidad orgánica o, si se quiere, la exteriorización de la inercia en la vida orgánica (p. 36).
\end{abstract}

En cuanto a la pulsión, se estaría ante un concepto realmente irreductible, mas, sin embargo, de ello se puede decir que es una energía constante y fluctuante, orientada hacia un objeto de quien se exige satisfacción. Por ello, la pulsión no cesa de insistir. 
Ante esto, se plantea que el sujeto adicto se encuentra gobernado y entregado a la pulsión de muerte y al exceso de un goce mortífero, el cual ligado al deseo exige satisfacción inmediata y, que el mismo sujeto intenta socorrer con la sustancia adictiva. El yo-corpóreo no encuentra salida airosa considerando que es el mismo cuerpo el que es exigido hasta su máxima expresión o máximo aguante. De esta manera, el adicto entregado a una investidura ligada por el proceso secundario al objeto de satisfacción, se encuentra cada vez más entregado a su propia autodestrucción. Respecto a ello, Freud (1920) expresa que:

Puesto que todas las mociones pulsionales afectan a los sistemas inconscientes, difícilmente sea una novedad decir que obedecen al proceso psíquico primario; y, por otra parte, de ahí a identificar al proceso psíquico primario con la investidura libremente móvil, y al proceso secundario con las alteraciones de la investidura ligada o tónica de Breuer, no hay más que un pequeño paso. Entonces, la tarea de los estratos superiores del aparato anímico sería ligar la excitación de las pulsiones que entra en operación en el proceso primario (pp. 34-35).

\section{MÉTODO}

El estudio se realizó bajo el paradigma postpositivista de la investigación cualitativa, utilizando una episteme hermenéutica para la interpretación de los significados en la historia de vida y, para la comprensión del alcoholismo como goce autoerótico propiciado por el sujeto adicto como manifestación sintomática.

Eldiseño delainvestigación fue planteado como una historia de vida del paradigma cualitativo de la investigación científica, tomando la orientación teórica psicoanalítica para la interpretación de la misma y generar conocimiento científico.
El método usado fue la historia de vida, desde este panorama, la investigación se tornó hacia la vivencia particular del sujeto como actor de ella, y donde el acercamiento desde la experiencia directa del investigador proporciona el encuentro con el sujeto, la historia y su razón de ser. Por otra parte, Moreno (1998), planteó que: “Me interesa la vivencia concreta de hombres reales y en particular cómo viven sus relaciones afectivas, esto es, sus vínculos de aproximación y alejamiento, las redes que con ellos tejen, el drama de su concreta libertad." (p. 185). Por ello, la investigación se direccionó hacia la historia misma de vida y no en datos empíricos, sin buscar otra cosa que lo que ella misma comunica.

La validez de los resultados en la investigación cualitativa se afianzó en la capacidad del investigador para adentrarse en el mundo vivido del historiador como actor del mismo y encontrar en ella el sentido más próximo a su realidad.

La fase que se abordó en la investigación fue prehistoria, historia e interpretación o comprensión.

En la fase prehistoria, el investigador tuvo que ahondar en el establecimiento de una fase previa a la historia, donde manifieste la relación existente entre el historiador y cohistoriador, como también el conocimiento previo que se obtuvo del historiador.

En la fase historia, se da inició a la elaboración de la historia de vida. De esta manera, el cohistoriador junto con el historiador, se sumergen en una dialéctica propiciadora de los sentidos inmersos dentro de las lagunas del lenguaje.

En la fase tercera o la interpretación, se dividió en dos partes, la primera consistió en el ordenamiento del discurso del historiador en huellas de sentido y la segunda, en la interpretación de los mismos bloques. 


\section{RESULTADOS}

Julio Luna, es un hombre divorciado y soltero, el cual, reside en Guacara, Estado Carabobo, en casa de sus padres y donde creció de niño. Julio vive con su madre y su padre extranjero, más específicamente italiano, quienes son de edad bastante avanzada. Julio estudió abogacía y actualmente es estudiante de psicología del octavo semestre en la universidad Arturo Michelena.

De igual manera, convive en casa con una de sus siete hermanas, donde residen los hijos de dicha hermana. Los demás hermanos de Julio conviven muy cercanos a él, considerando que tienen un terreno familiar amplio.

Dentro de esta dinámica familiar, la madre de Julio, se dedicaba al cuidado del hogar y algunas atenciones que amerita su esposo. Actualmente Julio, dedica la mayor parte de su tiempo al cultivo en los grandes espacios de su hogar y eventualmente al trabajo del ejercicio del derecho. De igual manera, dedica tiempo a la actual carrera de psicología en la cual lleva aproximadamente seis años, pero no ha logrado graduarse por el atraso que ha tenido en las reprobaciones.

Julio asumió haber tenido historia con el alcohol por lo que su vida se vio afectada significativamente.

En este sentido, Julio inició su relato de vida, recordando su infancia:

Mi historia de vida podría comenzar diciéndote que bueno nada, un muchacho hijo de extranjero, con mi mamá que es venezolana $y$, bueno, criado aquí en el toco, en una casa grande con un terreno donde siempre han abundado los árboles y las frutas $y$, bueno relativamente esa niñez que puedo recordar fue una niñez sana y feliz, llena de todos esos espacios que bueno para un niño, lo que es el campo y el espacio lo hace feliz.
Ya adentrado en la adolescencia empezaron a presentarse ciertos conflictos con su padre.

Ciertamente, bueno, como hijo de extranjero, siempre ha habido esa cultura muy fijada más que todo, por parte del viejo $y$, bueno, que simplemente hace una dinámica diferente ¿no?, que, en algún momento, al no entender muchas cosas, de repente puede decirse de que hay un choque ¿no?, y esto, te estoy hablando más que todo en la edad de la adolescencia, cuando ya uno entra en esa adolescencia, pienso yo, en esa búsqueda de personalidad como tal, de ¿Quién eres?, uno empieza bueno, a tener todas estas situaciones, por ejemplo, en mi caso que siendo mi papa una persona fuerte de carácter imagínate tú, siempre hubo esa parte de bueno, de ese carácter fuerte ¿no?, y, hoy en día lo entiendo, también no era que había una falta de amor ni de cariño, sino más bien una falta de saberlo manifestar ¿no? (...), cuando uno empieza a llegar a la adolescencia, uno empieza a tener digamos de alguna forma un conflicto, a nivel de esas relaciones entre mi papá, cosas de aquí mismo en la casa, mi mamá y, bueno, te estoy hablando de una época que más o menos estaba como en tercer año de bachillerato $y$, bueno, también con los factores de la presión, digamos de la presión del grupo, más que todo en bachillerato, fue allí donde comencé a tomar, recuerdo que fue en una fiesta del liceo, fuimos varios amigos $y$, bueno, fue la primera vez como digamos esa primera vez que me eché una borrachera, (...) que fue una experiencia que no me gusto, obviamente si fue algo placentero en el sentido de que por primera vez, todo ese conflicto familiar quedaba atrás ¿no?, toda esa búsqueda que daba como sosegada, entonces ¿Qué sucedió?, que bueno, las practicas fueron y se fue haciendo cada vez más frecuente el beber. 
Con el consumo de alcohol se empezó a intensificar las confrontaciones entre el papá y Julio, llegando a la violencia y maltrato, lo cual, generó más rebeldía y acercamiento a los grupos de amigos con quienes bebía alcohol,

(...) me di cuenta estaba bebiendo ya casi todos los días, ósea, cada vez más tenía la necesidad de estar en ese estado (...) tengo que darle freno de mano a esta vaina porque ¿A dónde voy a llegar? Claro, obviamente cuando hablamos de alcoholismo, muchas veces no sabemos distinguir y eso lo puedo ver hoy en día ¿no?, en ese tiempo, hubo, tal vez mucho sentimiento de culpa en el sentido de pensarte a ti mismo como un alcohólico ¿no?, que básicamente, como sustancia aceptable por la sociedad, incluso socialmente aceptable, obviamente hay una línea muy bizarra ¿no?, en saber cuándo eres un alcohólico y cuando no, y más que todo en esta cultura en el que el beber es una práctica que básicamente es normal.

Julio hizo una confesión sobre su padre y su influencia con el alcohol:

yo me acuerdo que mi papá también bebía y, claro, obviamente siempre nos sacaba con él para todos lados cuando iba a pescar, iba para la playa o cualquier sitio y, yo me acuerdo que yo era él, ¿Cómo se llama?, el copiloto de la vaina, le destapaba las cervezas y se las pasaba y, obviamente, tal vez lo que parece muy inocente en ese momento, se convierte el algo que bueno, estas adoctrinando algo, estas entrenando algo ahí ¿no?, eso creo que también lo veo mucho Wakkas, en lo que es la parte de la cerveza polar, que con el Maltín Polar van adoctrinando a los niños y a través del deporte, de los juegos de beisbol y todo $y$, cuando vienes a ver, no se toman la cerveza sino un Maltín pero ahí en alguna forma vas y agarras la cerveza.
Una vez, graduado de bachiller, Julio, se mudó a Canadá por cinco años, y el problema de alcoholismo continuo.

En Canadá pase cinco años, obviamente, al estar allá solo, un chamo también como de dieciocho o diecinueve años imagínate. Creo que es ahí en ese momento, donde realmente considero el buscar un poco de ayuda, algún tipo de ayuda, ¿Por qué?, porque estando en Canadá, me acuerdo que bueno, no tenía estos amigos, no tenía incluso ese seno familiar que de alguna u otra forma es un núcleo. Empecé a tomar en Canadá, a tomar y bueno, tomaba los fines de semana como te digo de una forma social, pero, después me vi hasta tomando solo, nada más por tomar nada más por tomar ¿no?, y el deseo de tomar no era ya el mismo deseo de simplemente disfrutar un rato de unos tragos, sino que, si podía beberme la media botella rápido de un solo golpe para llegar a estar medio prendido mejor, ósea, era ya buscar esa sensación, no era ya un aspecto social.

En Canadá, Julio estudio Derecho, sin embargo siempre estuvo el alcohol presente.

(...) y estudie derecho, obviamente siempre el alcohol a estado de alguna forma en mi vida porque bueno, hasta hoy en día todavía sigo tomando unos tragos pero, si te dijera de caer en un nivel de alcoholismo como tal, bueno no llego a suceder en mi pero si hubo los principios pues, de eso, hay que tener ojo con eso, ¿Por qué?, porque como te digo pues, ciertamente uno a veces justifica, incluso te engañas pensando que no eres un alcohólico y no lo aceptas ¿no?, pero eres alcohólico desde el momento que bebes, tal cual, desde el momento que tienes las ganas de beber (...) después me devolví a Venezuela. 
Julio comentó que en la actualidad no bebe nada, agradeciendo a Dios por eso,

bueno, como te digo, al decir nada es nada comparado a mis tiempos en que bebía ¿no?, que podía durar dos o tres días bebiendo y pegado en una sola bebezón con puros carajos y bueno, así, pero pienso que la situación económica también ha influido en gran parte obviamente ahorita no es la misma posibilidad de tu tomar todos los días ¿no?, y cuando tú te vas adecuando, simplemente como te digo, llega un momento que te adaptas a ese cambio.

\section{El alcohol como rebeldía}

es una forma de tu decirles a tus padres, o no decirles, de alguna forma de hacerles sentir también mal a ellos ¿no?, de hacerlos sentir mal porque sabes, estos flagelos, la droga, el alcohol, siempre te recalcan desde pequeño y como que siempre te hacen ver, o por lo menos en mi caso, que el tu caer en eso sería algo que los lastimaría mucho y los decepcionaría y, creo que en una forma inconsciente cuando llega esa parte rebelde que tú te enfrentas con esos padres $y$, obviamente con la imposibilidad de no poderlos golpear o hacerlos sufrir como tu sientes que ellos te están haciendo sufrir, entonces bueno, empiezas a tomar para de alguna forma, hacerlos sentir a ellos mal, pienso que es así.

Sin embargo, cuando Julio era adolescentes entre 12 a 13 años practicaba karate, y los vicios para él iba en contra de su disciplina.

yo era un atleta, nunca pensaba en el alcohol como algo bueno, lo que era el alcohol y el cigarrillo para mí era fatal pues, porque desde (...), mi gimnasio (...), veía eso y lo veía como algo que imagínate, ósea el fumar me restaba condiciones, el tomar me restaba condiciones y yo tenía que estar en óptimas condiciones.

Pero justo, los conflictos continuos entre su padre y él, encadenaron conductas que lo llevaron a probar el alcohol por primera vez,

Y luego, que me acuerdo que fue ese día que fue la primera vez que tome fue porque tuve en horas antes, un problema con el viejo que bueno, eso fue horrible, me dio con un tubo por la cabeza y llegamos bueno a un encontronazo que yo le lance una silla, que no le pegue ¿no?, pero si se la lance para darle. Entonces creo que desde ahí, que salí de aquí con toda la rabia, (...), todo movido, bueno me encontré este grupo de panas del liceo y llegamos a un apartamento de un pana que no estaba la mamá y empezamos a tomar y bueno yo tome más como para bueno, para de alguna forma darle palo a ese problema que tenía (...) y bueno me acuerdo que me tome el primero y no me gusto, y dije: ¿cómo puede tomar esto?, jestán locos!, ¡esto es como tomar veneno!, ¿cómo vas a decir que esto es placentero?, me acuerdo clarito, entonces es mejor tomarse un jugo, una malta pensaba yo, ¡que locura es esta!, y bueno, después del cuarto que se puso buena, ¿Por qué?, porque después del cuarto ya no sabía así tan amargo, ni tan fuerte ni nada así, sino que más bien empecé a sentirme como más relajado, empecé a sentirme más alegre, más feliz pues, más conforme y, también, mentalmente también empecé a sentirme con menos sentimientos de culpa, más justificado, pensaba que bueno, él también se lo mereció porque si a mí me tratan así yo tengo que tratar igual $y$, desde ese momento dije: nada, voy a decidir ser así, si tú me das amor te doy amor y si me das palo te doy palo y así de alguna forma creí que era lo más justo. 
Esto influyó mucho en el deporte de Julio, hasta el punto de ser expulsado de la asociación de karate, ya que en varias ocasiones llegó en estado de ebriedad grave.

(...) más nunca fui al karate, más nunca hasta ahora, más nunca toque nada de lo que tiene que ver con eso, ósea, fue como que si lo borre, igual que con Canadá, después que me vine de Canadá ¿sabes?, lo borre de mi vida que incluso hasta el inglés y todo $y$, a pesar de que yo se hablar inglés y bueno, lo se hablar bien, nunca... no me gusta, es algo que ¿sabes?, trato siempre de... no sé, de poner en una sombra (...), que lo guardo ahí, porque te digo, el karate y muchas otras cosas que fueron talento en alguna vez de mi vida, fueron talentos que tuve, como dibujar, yo dibujo bien, bien $y$, nunca más dibuje tampoco (...), después de eso tuve ese cambio radical, ósea, me refugie más en el alcohol.

\section{Bloque-de-Sentido: El alcohol y el autoerotismo}

La historia de Julio se direcciona hacia las sensaciones logradas a partir de la ingesta de la sustancia embriagadora. A partir de las primeras sensaciones encontradas en los inicios de consumo de Julio, el alcohol tomó un significado de mayor valor y se volvió una incesante búsqueda del placer asociado al consumo. En ello surge la marca-guía: la búsqueda incesante de la ebriedad.

Es entonces para Julio, el encuentro con un goce que le garantiza placer en el displacer, donde se permitió alcanzar esas sensaciones añoradas de su prehistoria anímica pero sepultadas, a partir de la castración que no toleró. Es por ello, que la dependencia hacia la sustancia se tornó, tal que sin ella, solo quedaría el desconcierto y el dolor que lo acompañaría. Surge de esta manera la marca guía: autoerotismo y el sepultamiento del padre.
Todo se reduce a la sustancia embriagadora como goce mortífero sentido como displacer en el yo, pero como placer en la economía libidinal asociado a la satisfacción pulsional. Esto puede verse en la historia de Julio, cuando el mismo se da cuenta de las sensaciones alcanzadas en su ingesta a pesar de las consecuencias que le deparaba el consumo del mismo. En ello surge, la marca guía: el alcoholismo y el encuentro con las sensaciones añoradas de la primera infancia.

De esta manera, fue a través del alcohol que Julio llegó a un éxtasis donde encontró desde la fantasía, el poder de renegar aquello que lo avergonzaba y que lo limitaba. Es entonces, con el alcohol donde el mismo Julio, tomó el control sobre aquello que lo gobernaba. Por todo esto, surge la marca-guía: el enaltecimiento del objeto alcohol.

\section{CONCLUSIONES}

El alcohol en la vivencia del sujeto adicto concierne al hecho de que la sustancia se posiciona en la vida del adicto como un objeto que garantiza el encuentro con el erotismo primitivo de la primera infancia. Por ende, ingerir la bebida embriagadora significa propiciar en su mismo cuerpo las sensaciones de satisfacción sexual primitiva y con ello, la masturbación como adicción primordial queda relegada por el objeto alcohol. Desde este panorama, el sujeto adicto se encuentra sumergido en un ser para sentir desde la ingesta de alcohol y las secuelas que depara el consumo del mismo.

El alcohol, es el encuentro con un goce autoerótico que soslaya la falta inmersa del ser y, por ende, el alcoholismo como síntoma adquiere forma y relevancia a partir de este goce mortífero 
del cual se entrega el adicto sin medidas. Todo esto, se direcciona hacia centralizar el goce autoerótico como protagonista del mantenimiento del síntoma por las sensaciones encontradas en esta forma de gozar desde la fantasía yoica con el objeto alcohol.

El significado existente del objeto alcohol en la vida del historiador, cumple con la función de propiciar en su propio cuerpo una forma primitiva de satisfacción erótica para así gozar de ello sin pasar por el goce fálico, que implica relacionarse con el otro a partir de la relación sexual. En el historiador se hizo evidente como cuando por ejemplo argumenta: ingerir la sustancia para sentir las sensaciones o para llegar al éxtasis que le propiciaba, ingerir la sustancia para escapar del conflicto con la severidad del padre, ingerir la sustancia para sentirse justificado, ingerir la sustancia para encontrarse con las sensaciones de superioridad, la preferencia por la sustancia respecto a las actividades que exigen relacionarse. Todo ello, denota el significado del alcohol en las prácticas de vida del historiador.

Para finalizar, la soledad manifestada en Julio Luna, es una de las vertientes que llevo al investigador a vislumbrar en este actor la posibilidad de sumergirse junto al investigador en el sin fin de significados que implica la experiencia. Durante su historia, se puede ver en él la imposibilidad de sostener un deseo de permanencia en el lazo social, el goce toma más bien vías alternas o donde no sea necesaria la comunión entre personas. Por otra parte, la inconstancia respecto a sus labores lo llevaba a sumergirse cada vez más en este goce, donde conseguía aplazar el sufrimiento.

\section{REFERENCIAS}

Cifuentes, A. (2014). De arrebatos y adicciones: consideraciones psicoanalíticas sobre la clínica de lo femenino en el campo de las toxicomanías. Santiago, Chile
Cifuentes, A. (2015). Adicciones en mujeres: del estrago materno (Ravage) al arrebato femenino (Ravissement): Revista psicológica Praxis. Santiago, Chile

Diaz, M. (2017). Venezuela tiene la tasa más alta de muertes asociadas al alcohol: El Clarín. Disponible en http://elclarinweb.com/ exclusiva-el- clarín/Venezuela-la-tasa-masalta-muertes-asociadas-al-alcohol

Freud, S. (1893/1899). Primeras publicaciones psicoanalíticas: La sexualidad en la etiología de las neurosis. En J.L Etcheverry (Esd.), Obras completas: Sigmund Freud (vol. 3). Buenos Aires, Argentina: Amorrortu Editores (1991)

Freud, S. (1916/17). Conferencias de introducción al psicoanálisis (Parte III): Conferencia. El sentido de los síntomas. En J.L Etcheverry (Esd.), Obras completas: Sigmund Freud (vol. 16). Buenos Aires, Argentina: Amorrortu Editores (1991)

Freud, S. (1920/1922). Más allá del principio de placer, Psicología de las masas y análisis del yo y otras obras: Más allá del principio de placer. En J.L Etcheverry (Esd.), Obras completas: Sigmund Freud (vol. 18). Buenos Aires, Argentina: Amorrortu Editores (1992)

Lacan, J. (1938). La familia: El complejo, factor concreto de la psicología familiar. En Víctor Fishman (Eds). Buenos aires (2010). Editorial Argonauta

Lacan, J. (1972/73). Seminario 20. Aun: Del goce. Buenos aires. Paidós

Lora, M., y Calderón, C. (2010). Un Abordaje a la toxicomanía desde el psicoanálisis: Sistema de Información Científica Redalyc. La paz, Bolivia

Miller, J. (1989). Para una investigación sobre el goce autoerótico. Revista Pharmakon digital (2015)

Mora, H. (2015). El goce en la toxicomanía: Blog de la Nueva Escuela Lacaniana Maracaibo. Maracaibo, Venezuela

Moreno, A.; Brandt, J.; Campos, A.; Navarro, R.; Pérez, M.; Rodríguez, W., y Valera, Y. (1998). Historia de vida de Felicia Valera. Caracas, Venezuela: Fondo Editorial CONICIT

Pasteur, L. (1880). De l'attbnuation du virus du chokra des poules. Comptes rendus de l'Academie des sciences, 26 October 1880, Vol. 91, pages 673-680 
Rojas, A.; Ceballos, C., y Gaspard, J. (2007). Actualidad de una clínica del a-sujeto. El ejemplo de la toxicomanía: Revista de psicoanálisis. Bogotá, Colombia
Sarmiento, R.; Carbonell, I.; Plasencia, C., y Ducónger, R. (2011). Patrones de consumo de alcohol en una población masculina de El Caney: Revista Medisan Santiago de Cuba, Cuba 